OPEN ACCESS

Edited by:

Maria Dolores Ledesma,

Severo Ochoa Molecular Biology

Center (CSIC-UAM), Spain

Reviewed by:

Margaret Ellen Flanagan,

University of Minnesota Twin Cities,

United States

Elisabetta Albi

Università Degli Studi Di Perugia, Italy

*Correspondence:

Nady Braidy

n.braidy@unsw.edu.au

Specialty section

This article was submitted to

Dementia,

a section of the journal

Frontiers in Neurology

Received: 05 March 2019

Accepted: 29 July 2019

Published: 21 August 2019

Citation:

Wong MWK, Braidy N, Pickford R, Sachdev PS and Poljak A (2019) Comparison of Single Phase and Biphasic Extraction Protocols for Lipidomic Studies Using Human Plasma. Front. Neurol. 10:879. doi: 10.3389/fneur.2019.00879

\section{Comparison of Single Phase and Biphasic Extraction Protocols for Lipidomic Studies Using Human Plasma}

\author{
Matthew Wai Kin Wong ${ }^{1}$, Nady Braidy ${ }^{1 *}$, Russell Pickford ${ }^{2}$, Perminder Singh Sachdev ${ }^{1,3}$ \\ and Anne Poljak ${ }^{1,2,4}$
}

${ }^{1}$ Centre for Healthy Brain Ageing, School of Psychiatry, Faculty of Medicine, University of New South Wales, Sydney, NSW, Australia, ${ }^{2}$ Bioanalytical Mass Spectrometry Facility, University of New South Wales, Sydney, NSW, Australia, ${ }^{3}$ Euroa Centre, Prince of Wales Hospital, Neuropsychiatric Institute, Sydney, NSW, Australia, ${ }^{4}$ School of Medical Sciences, Faculty of Medicine, University of New South Wales, Sydney, NSW, Australia

Lipidomic profiling of plasma is an emerging field, given the importance of lipids in major cellular pathways, and is dependent on efficient lipid extraction protocols. Recent attention has turned to plasma lipidomics as a means to identify potential diagnostic and prognostic biomarkers related to dementia, neuropsychiatric health and disease. Although several solvent-based lipid extraction protocols have been developed and are currently in use, novel and more efficient methods could greatly simplify lipid analysis in plasma and warrant investigation. Human plasma from normolipidemic adult volunteers was collected to evaluate three different solvent extraction protocols, including the classical Folch method, the methanol/tert-butyl methyl ether (MTBE) (Matyash) method, and a recent single-phase methanol/1-butanol (Alshehry) method. Extracted lipids were analyzed using liquid chromatography mass spectrometry (LC-MS) in positive and negative ion mode. Overall, more than 500 different lipids were identified in positive and negative ion mode combined. Our data show that the single phase Alshehry method was as effective as the Folch and Matyash methods in extracting most lipid classes and was more effective in extraction of polar lipids. Normalized peak areas of the Alshehry method were highly and positively correlated with both the Folch and Matyash methods $\left(r^{2}=0.99\right.$ and 0.97, respectively). Within- and between- subject correlations were $r=0.99$ and 0.96 , respectively. Median intra-assay coefficient of variation (CV\%) in positive mode was 14.1, 15.1, and 21.8 for the Alshehry, Folch and Matyash methods, respectively. Median Alshehry inter-assay CV (collected over 5 separate days) was 14.4\%. In conclusion, the novel Alshehry method was at least as good as, if not better than the established biphasic extraction methods in detecting a wide range of lipid classes, using as little as $10 \mu \mathrm{L}$ of plasma, and was highly reproducible, safer and more environmentally-friendly as it doesn't require chloroform.

Keywords: lipidomics, lipid extraction, mass spectrometry, plasma lipids, chromatography 


\section{INTRODUCTION}

It is well-documented that lipids have multiple structural and functional roles, including signaling $(1,2)$, maintenance of membrane structure $(3,4)$, myelin sheath formation $(5,6)$, neurotransmission (7), and protein interactions in both plasma and organs including the brain $(8,9)$. Physiological processes such as synaptic and mitochondrial function, and lipid raft formation are critically dependent on lipid composition (1012). By contrast, lipid by-products, particularly metabolites of arachidonic acid and lipid products of oxidative stress are drivers of inflammation (13). Consequently, the plasma lipidome has a considerable impact on the cellular lipid environment, vasculature function, and inflammatory and oxidative processes. The plasma lipid profile therefore also represents a lifestyle modifiable factor which can play a decisive role in the health state and maintenance of cognitive function during aging (14).

The field of lipidomics is constantly expanding, enabling high throughput analysis of lipid analytes in crude extracts for the study of health and disease. Recent advances in mass spectrometry have resulted in greater sensitivity, increased mass accuracy and faster scan speeds $(15,16)$. This has enabled greater sensitivity and better characterization of lipid changes in bodily fluids, cells, and tissue extracts, leading to renewed understanding of the role of different lipid classes in the pathobiology of diseases. Lipidomics has recently been applied to the study of dementia, where brain, CSF and plasma lipids have been identified as potential diagnostic and prognostic biomarkers for Alzheimer's disease $(17,18)$, frontotemporal dementia (19), as well as other common neuropsychiatric disorders, such as schizophrenia and bipolar disorder $(20,21)$.

The future of lipidomics lies in clinical application. As such, blood-based biomarkers are ideal since venepuncture is already routinely used in the clinic and is less invasive compared to CSF extraction via lumbar puncture. Additionally, it is much simpler to take repeat measurements of blood which may be useful for longitudinal analysis. We will discuss further the relationship of centrally derived biological signals with the periphery and vice versa in this paper. Although interest in lipidomics especially in the study of health and disease is expanding, there is still much room for improvement in lipidomic methodologies for research and clinical use. Since lipidomics often involves the study of 100 to 1,000 of individual biological samples within a single experiment, any improvement to the cost or efficiency of sample processing could have a considerable impact on streamlining plasma lipidomics research.

One key area in which improvement is possible is in the extraction process, whereby total lipids within plasma, tissue, and other biological extracts are isolated prior to further analysis. Solvent-based extraction systems must efficiently extract lipids present in biological samples while minimizing bias, lipid degradation/oxidation, or contamination from nonlipid components, such as sugars, peptides and amino acids. Therefore, effective and reliable identification and profiling of lipids in plasma is dependent on the efficiency of the lipid extraction protocol (22). The traditional Folch method (2:1 chloroform/methanol) (23), developed in 1957 is still used as a benchmark extraction process in many laboratories for a wide range of biological fluids, including, blood, tears, urine, saliva, cerebrospinal fluid, human milk, bronchoalveolar lavage fluid and sperm (22). The Folch method is based on the partitioning of lipids in a biphasic mixture of chloroform and methanol. Methanol disrupts hydrogen bonds between lipids and protein following addition of an organic solvent such as chloroform.

Alternative methods, such as the Matyash (tert-butyl methyl ether (MTBE)/methanol) (24), have gained popularity, in particular for the extraction of sphingolipids. Replacement of chloroform with MTBE demonstrated similar extraction of major lipid classes $(22,24)$. Recently, a single phase (1:1 1butanol/methanol) extraction developed by Alshehry et al. (25, 26) has also been published. Both newer methods eliminate the need to draw lipids from the lower phase, thereby saving time compared to the Folch method, and are safer and more environmentally friendly as they do not use toxic chloroform. Moreover, the Alshehry method does not involve any biphasic solvent separation and could therefore be even more convenient, and potentially yield better lipid recoveries, than the Matyash method. The Matyash method has already been shown by independent laboratories to be as good, if not better, at extracting lipids as the Folch method $(22,24,27)$. A recent comparative study of multiphasic methods has been published (28), however the single phase Alshehry method was only recently published and has not been compared with established biphasic methods.

In this paper, we examine the performance of the singlephase Alshehry extraction system and compare the results against the two biphasic extraction methods, the Matyash method and the traditional Folch method (Figure 1) in terms of coverage of lipids extracted and reproducibility from pooled plasma of healthy volunteers. Liquid chromatography mass spectrometry (LC-MS/MS) was used to identify the lipids extracted.

\section{METHODS}

\section{Reagents and Chemicals}

HPLC grade methanol was purchased from Thermo Fisher Scientific (Sydney, Australia). Analytical reagent grade 1-butanol was purchased from Asia Pacific Specialty Chemicals (APS) (Sydney, Australia). Other high grade solvents and reagents were used, including MTBE (Sigma Aldrich, Israel), chloroform (Honeywell, USA), acetonitrile (Honeywell, Korea), formic acid (Chem-supply, Australia), ammonium formate (Honeywell, Germany). Isopropanol was of LC-MS grade (Honeywell, Michigan, USA). SPLASH Lipidomix stable isotope labeled internal standards were purchased from Avanti (Alabaster, Alabama, USA). All other reagents were analytical reagent grade. MilliQ water was used for buffer preparation and had a minimum resistivity of $18 \mathrm{~m} \Omega$.

\section{Internal Standards}

Internal standards were purchased from Avanti (Splash Lipidomix, Alabaster, United States) and included phosphatidylcholine 15:0-18:1(d7) PC, phosphatidylethanolamine 15:0-18:1(d7) PE, phosphatidylserine 15:0-18:1(d7) PS, phosphatidylglycerol 15:0-18:1(d7) PG, 


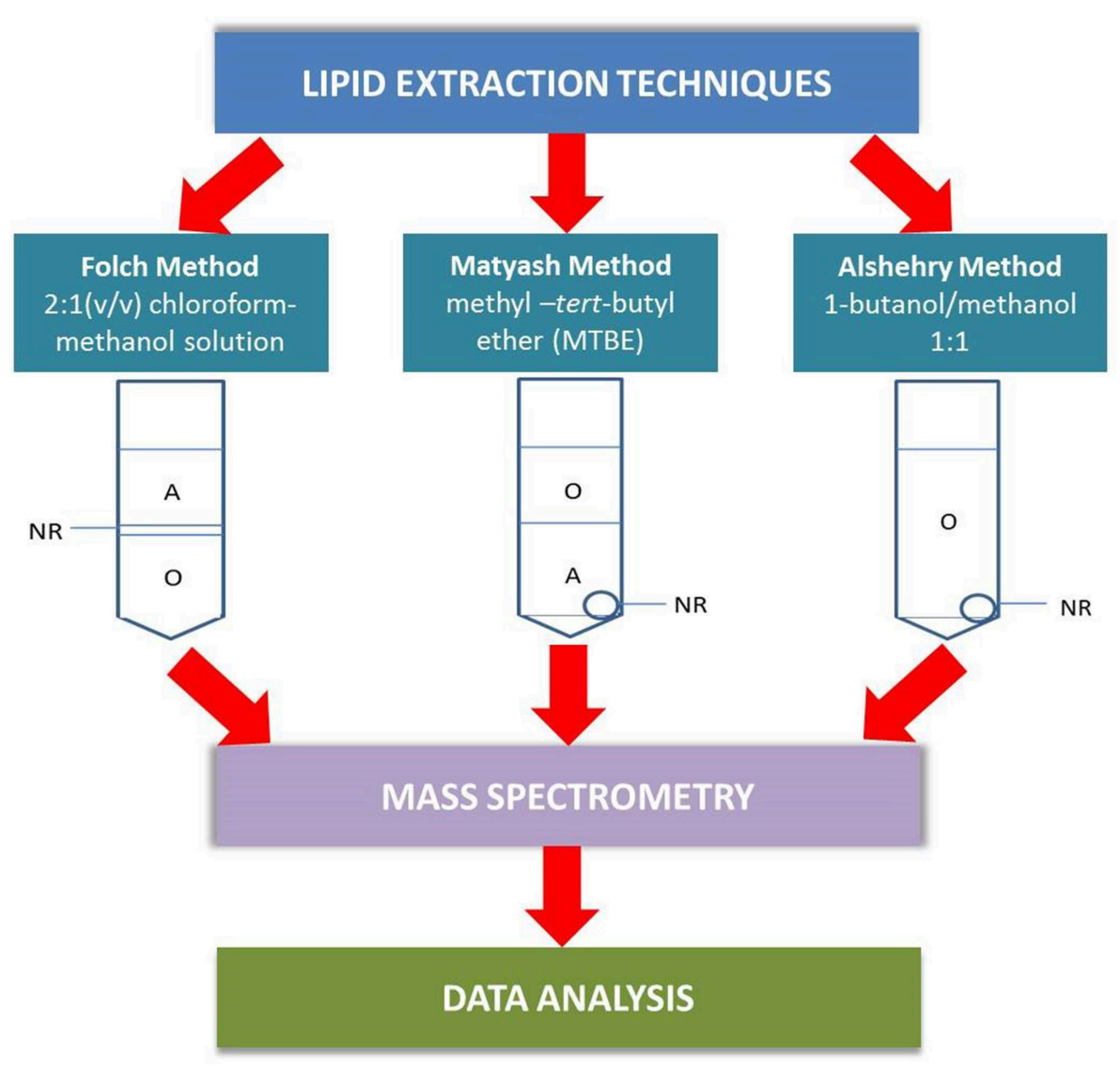

FIGURE 1 | A schematic outlining the differences between the plasma extraction methods in the analytical pathway. In the Folch method, the lipid-containing organic phase $(\mathrm{O})$ lies beneath a non-extractable residue (NR) and must be collected by passing a micropipette through the aqueous phase (A) and NR. The Matyash MTBE extraction simplifies the extraction process by having the lipids collected in the upper phase, though care must be taken not to take up the aqueous phase. The Alshehry 1-butanol/methanol extraction collects all lipids within a single-phase supernatant, with NR as a white pellet at the bottom of the tube.

phosphatidylinositol 15:0-18:1(d7), PI, phosphatidic acid 15:0-18:1(d7) PA, lysophosphatidylcholine 18:1(d7) LPC, lysophosphatidylethanolamine 18:1(d7) LPE, cholesteryl ester 18:1(d7) CE, monoacylglycerol 18:1(d7) MG, diacylglycerol 15:0-18:1(d7) DG, triacylglycerol 15:0-18:1(d7)-15:0 TG, sphingomyelin 18:1(d9) SM and Cholesterol (d7). The Splash Lipidomix standards are deuterium labeled and not present endogenously, but with concentrations similar to physiological plasma concentrations of lipids for each class and thus are ideal for use as internal standards. A constant amount of internal standards was added to all samples and controls prior to lipid extraction $(10 \mu \mathrm{L}$ of a 1:10 dilution of SPLASH Lipidomix to $10 \mu \mathrm{L}$ neat plasma) for normalization of raw peak areas and to correct for differences in extraction and ionization efficiencies and matrix effects (29).

\section{Blood Collection}

Our study utilized fasting EDTA plasma collected from 10 cognitively "healthy" subjects aged between 65 and 85 years with informed consent, as part of a larger population-based study known as Sydney Memory and Aging Study (MAS) (30, 31).
Subjects did not have any forms of mild cognitive impairment or dementia, as assessed by a panel of neuropsychiatrists according to consensus diagnosis criteria $(30,32)$, All subjects had minimental state examination (MMSE) scores $>24$ (33) and no history of cardiovascular complications or diabetes mellitus. Plasma taken from the 10 subjects (five males and five females) were then pooled and aliquoted $(10 \mu \mathrm{L})$. Extractions from 10 pooled plasma aliquots were used to assess the intraassay coefficient of variation (CV\%) of each extraction method detailed below, while extractions taken on 5 separate days were used to assess inter-assay CV\% of the Alshehry method. Blood collection, processing and storage were performed under strict conditions to minimize preanalytical variability $(18,34)$, which included restricting plasma collection and aliquot to freezer time to a maximum of $2 \mathrm{~h}$, using EDTA plasma, and minimizing the number of freeze-thaw cycles. In particular, freezer storage of plasma and extracted lipids at $-80^{\circ} \mathrm{C}$ prior to analysis and limiting the number of freeze thaw cycles, is now part of international standardization guidelines in order to maximize stability of lipids over prolonged periods of time $(18,35,36)$. 
The study was approved by the Ethics Committees of the University of New South Wales and the South Eastern Sydney and Illawarra Area Health Service (ethics approval HC12313 and HC14327, respectively). All work involving human subjects conformed to the principles of the Declaration of Helsinki of the World Medical Association.

\section{Lipid Extraction Protocols Single Phase 1-Butanol/Methanol 1:1 (v/v) Alshehry Extraction Method}

Lipids were extracted as previously described (25, 26). Briefly, we added $10 \mu \mathrm{L}$ of internal lipid standards (Avanti SPLASH Lipidomix) that had been first diluted 1:10 in 1butanol/methanol, to $10 \mu \mathrm{L}$ aliquots of plasma in $0.5 \mathrm{~mL}$ polypropylene tubes (Eppendorf). 1-Butanol/methanol (100 mL, $1: 1 \mathrm{v} / \mathrm{v}$ ) containing $5 \mathrm{mM}$ ammonium formate was then added to the sample, vortexed $(10 \mathrm{~s})$, then sonicated $(1 \mathrm{~h})$. Tubes were centrifuged $(13,000 \times \mathrm{g}, 10 \mathrm{~min})$ and the supernatant removed into a clean polypropylene tube. A further $100 \mu \mathrm{l}$ of 1-butanol/methanol (1:1 v/v) was added to the pellet to re-extract any remaining lipids. The combined supernatant was evaporated by vacuum centrifugation and stored at $-80^{\circ} \mathrm{C}$ prior to analysis by LC-ESI MS/MS.

\section{Traditional Biphasic 2:1 Chloroform/Methanol (v/v) Folch Extraction Method}

Lipids were extracted as previously described (23), but using a 20fold scale down of volumes used in order to produce comparable results against the same volume of plasma as used in the Alshehry method. Briefly, $10 \mu \mathrm{L}$ aliquots of plasma and $10 \mu \mathrm{L}$ of internal lipid standards (Avanti SPLASH Lipidomix) that had been first diluted 1:10 in 1-butanol/methanol were added to $160 \mu \mathrm{l}$ of ice-cold methanol followed by addition of $320 \mu \mathrm{l}$ of ice-cold chloroform and vortexed $(10 \mathrm{~s})$, then sonicated $(1 \mathrm{~h})$. Tubes were centrifuged at $10,000 \times \mathrm{g}(10 \mathrm{~min})$. The upper aqueous phase was transferred into a fresh polypropylene tube and re-extracted by addition of $250 \mu \mathrm{l}$ ice-cold chloroform/methanol as described above. The upper phase was discarded, and the organic phase combined from both extractions, which was then evaporated by vacuum centrifugation and stored at $-80^{\circ} \mathrm{C}$ prior to analysis by LC-ESI MS/MS.

\section{Methanol-Tert-Butyl Methyl Ether Matyash Extraction Method}

Lipids were extracted as previously described (24) with a 20fold scale down of volumes; $10 \mu \mathrm{L}$ aliquots of plasma and 10 $\mu \mathrm{L}$ of internal lipid standards (Avanti SPLASH Lipidomix) that had been first diluted 1:10 in 1-butanol/methanol were added to $400 \mu \mathrm{l}$ of ice-cold methanol followed by addition of $500 \mu \mathrm{l}$ of MTBE, vortexed (10 s), then sonicated ( $1 \mathrm{~h}$ ). Afterwards, 500 $\mu l$ of MilliQ water was added to induce phase-separation. Tubes were centrifuged $(10,000 \times \mathrm{g}, 10 \mathrm{~min})$. The upper aqueous phase was transferred into a clean polypropylene tube and re-extracted by addition of $200 \mu \mathrm{l}$ MTBE as described above. The upper phase was discarded, and the organic phase was evaporated by vacuum centrifugation and stored at $-80^{\circ} \mathrm{C}$ prior to analysis.

\section{LC-MS Analysis}

Extracted lipid samples and lipid internal standards (Avanti SPLASH Lipidomix) were removed from the $-80^{\circ} \mathrm{C}$ freezer and resuspended in $100 \mu \mathrm{l}$ of 1 -butanol/methanol $(1: 1 \mathrm{v} / \mathrm{v})$ containing $5 \mathrm{mM}$ ammonium formate and transferred into Chromacol autosampler vials containing a $300 \mu \mathrm{l}$ glass insert. Lipid analysis was performed by LC ESI-MS/MS (Dionex LC system in-line to a Thermo QExactive Plus Orbitrap mass spectrometer; ThermoFisher Scientific; Waltham, Massachusetts). A Waters ACQUITY UPLC CSHTM C18 $1.7 \mu \mathrm{m}, 2.1 \times 100 \mathrm{~mm}$ column was used at a flow rate of 260 $\mu \mathrm{L} / \mathrm{min}$, using the following gradient: 32 to $100 \%$ solvent $\mathrm{B}$ over $25 \mathrm{~min}$, a return to $32 \% \mathrm{~B}$ and finally isocratic $32 \% \mathrm{~B}$ ( $5 \mathrm{~min}$ ) prior to the next injection. Solvents A and B consisted of acetonitrile: MilliQ water $(6: 4 \mathrm{v} / \mathrm{v})$ and isopropanol: acetonitrile $(9: 1 \mathrm{v} / \mathrm{v})$ respectively, both containing $10 \mathrm{mM}$ ammonium formate and $0.1 \%$ formic acid. The first $3 \mathrm{~min}$ of eluent, containing sample salts, was diverted to waste. Product ion scans in positive and negative ion mode were performed on each sample, to maximize numbers of lipid species identified. Sampling order was randomized prior to analysis. LC-MS grade isopropanol was used to minimize intensity of the background signal.

\section{Alignment and Peak Detection Using LipidSearch 4.2.2}

The raw data was aligned, and chromatographic peaks selected using LipidSearch software version 4.2.2 (Thermo Scientific, Tokyo, Japan), the database which now includes masses of the stable isotope labeled SPLASH Lipidomix internal standards. We performed search on raw files using the databases "General" and "labeled standards." For peak detection, recalc isotope was set to "ON," RT interval $=0.0 \mathrm{~min}$. We used product search for LC-MS method and the precursor and product tolerances were set at 5.0 and $8.0 \mathrm{ppm}$, respectively. The intensity threshold was $1 \%$ parent ion, and the $\mathrm{m}$-score threshold was set to 2.0 . For quantitation, $\mathrm{m} / \mathrm{z}$ tolerance was set at -5.0 to $5.0 \mathrm{ppm}$, and the retention time range was set at -0.5 to $0.5 \mathrm{~min}$. The $\mathrm{m}$-score threshold was 5.0, and all lipid classes were selected for inclusion. Ion adducts included $+\mathrm{H},+\mathrm{NH} 4$ for positive ion mode and $-\mathrm{H},+\mathrm{HCOO}$ for negative ion mode.

\section{Data Analysis}

The results from LipidSearch 4.2.2 were exported as a.csv file and opened in Excel software for further data processing and analysis. Peak areas of lipids in samples were normalized by dividing with peak area of the corresponding internal standard for that lipid class (i.e., peak area ratios). Correlations between normalized peak areas from the three extraction methods were calculated using the SPSS statistics software. The intra-assay coefficient of variation $(\mathrm{CV})$ was calculated by dividing the standard deviation of the normalized abundances of each lipid (for each method) by the mean across lipid species. Lipid ion identifications were filtered using the LipidSearch parameters $r e j=0$ and average peak quality $>0.75$. Recovery was calculated as the average raw intensity of internal standards spiked before extraction and divided by the average raw intensity of internal standards spiked post extraction. The inter-assay CV\% for the Alshehry 
method was also determined by comparing measurements from extractions taken across 5 separate days within a month.

\section{RESULTS}

\section{Concordance Between Methods}

When we compared the coverage of the different lipid classes based on extracted lipid internal standard peak areas, normalized peak areas in both positive and negative modes were highly and significantly correlated with the biphasic Matyash and Folch methods $(r=0.97$ and 0.99 respectively, $p<0.0001$, Figures 2A,B). This concordance was also reflected when comparing lipid measurements between duplicate extractions of plasma lipids prepared using the Alshehry protocol $\left(r^{2}\right.$ $=0.995)$, and in two different subjects, which is indicative of high reproducibility (Figures 2C,D). Additionally, BlandAltman analyses revealed that the Alshehry method largely agreed with the Folch and Matyash methods (bias ratio=1.09 and 1.247, respectively), except more for some lowly abundant peak areas, which were higher using the Alshehry method. All three methods produced similar number of LipidSearch molecular ion IDs prior to filtering $(381,400$, and 390 IDs in positive ion mode for the Matyash, Folch and Alshehry methods respectively, and 122,127 , and 119 IDs in negative ion mode, similarly).

\section{Comparison of Lipid Abundances}

One-way ANOVA was used to test whether the raw peak areas for internal standards using the Alshehry method were significantly different from the Matyash or Folch methods. There were no significant differences in peak areas between extraction methods for DG, PE and SM species ( $p>0.05)$. Peak areas of LPC, LPE, PI, PC and PG were higher in the Alshehry method than the Matyash method ( $p<0.05$, Tukey's post-hoc test), while the peak area of PG and PI were also greater in the Alshehry method relative to the Folch extraction. The Alshehry method was more efficient in extracting the highly polar lipids, such as LPC and LPE internal standard relative to the Matyash method ( $p<0.05$, one-way ANOVA, Tukey's post-hoc test) (Figure 3A).

Interestingly, TG was significantly higher in the Folch method compared to Matyash ( $p<0.05$, Tukey's post-hoc test), but yielded comparable, or slightly higher peak areas compared to Alshehry method, which did not reach significance $(p=0.08)$. Similarly, Alshehry peak areas of internal standards were larger than or equal to the Folch, and larger than the Matyash method in negative ion mode (Figure 3B, all $p<0.05$, Tukey's post-hoc test). Overall, the Alshehry method produces similar, if not greater yields than either the Folch or Matyash method.

\section{Recovery of Internal Standards}

Recoveries for each method were assessed by comparing the ratio of peak areas of internal standards spiked prior to and after extraction (Figure 4). The average recoveries were 99, 86, and $73 \%$ for the Alshehry, Folch and Matyash extractions, respectively. The highest recoveries for the Alshehry method were achieved with the phospholipids (>95\%) and lowest for the less polar TG, DG lipids (<80\%).
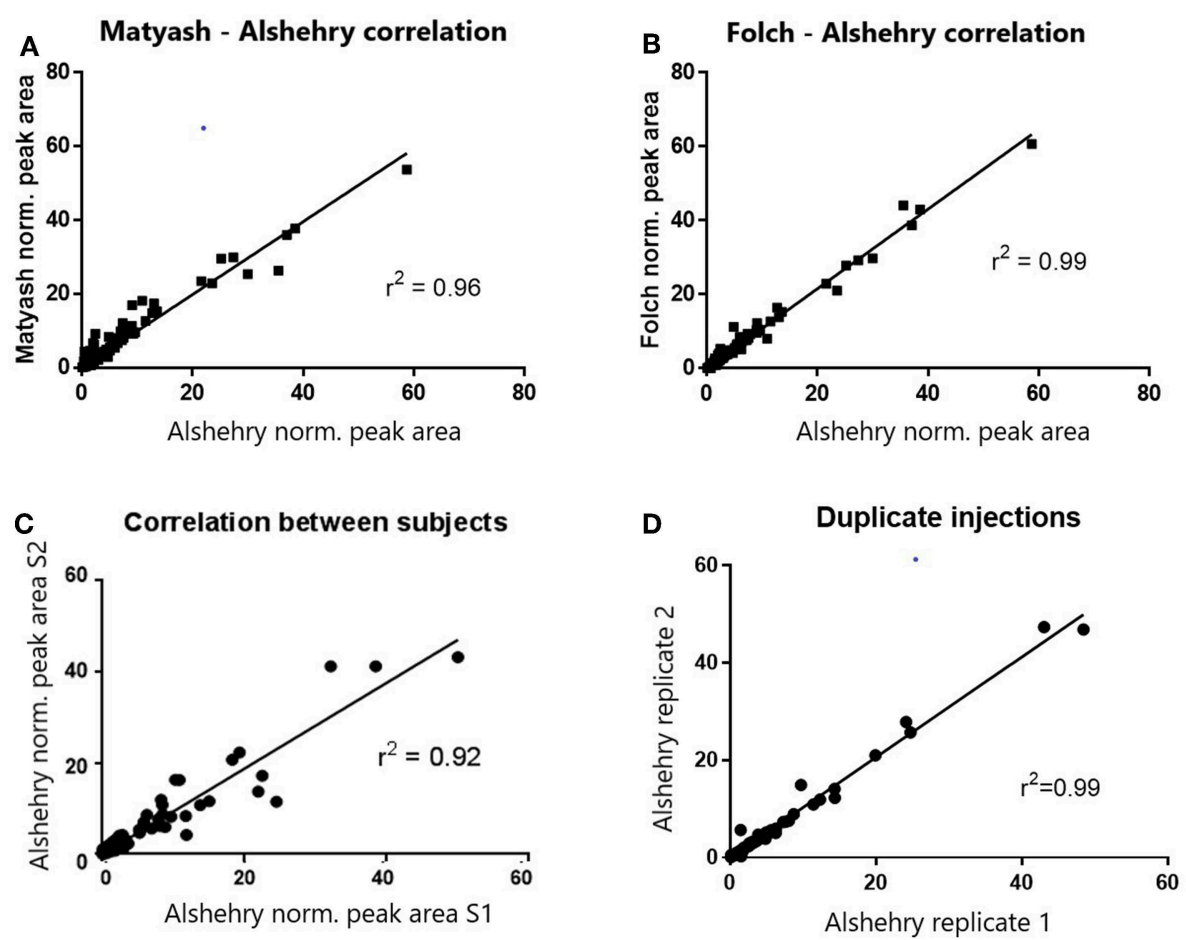

FIGURE 2 | Correlations of normalized peak areas between methods (A,B), subjects (C) and runs (D). Normalized peak areas of the Alshehry method were highly and positively correlated with both the Folch and Matyash methods. Within-subject correlation across separate runs was also high, though between- subject correlation was lower. 


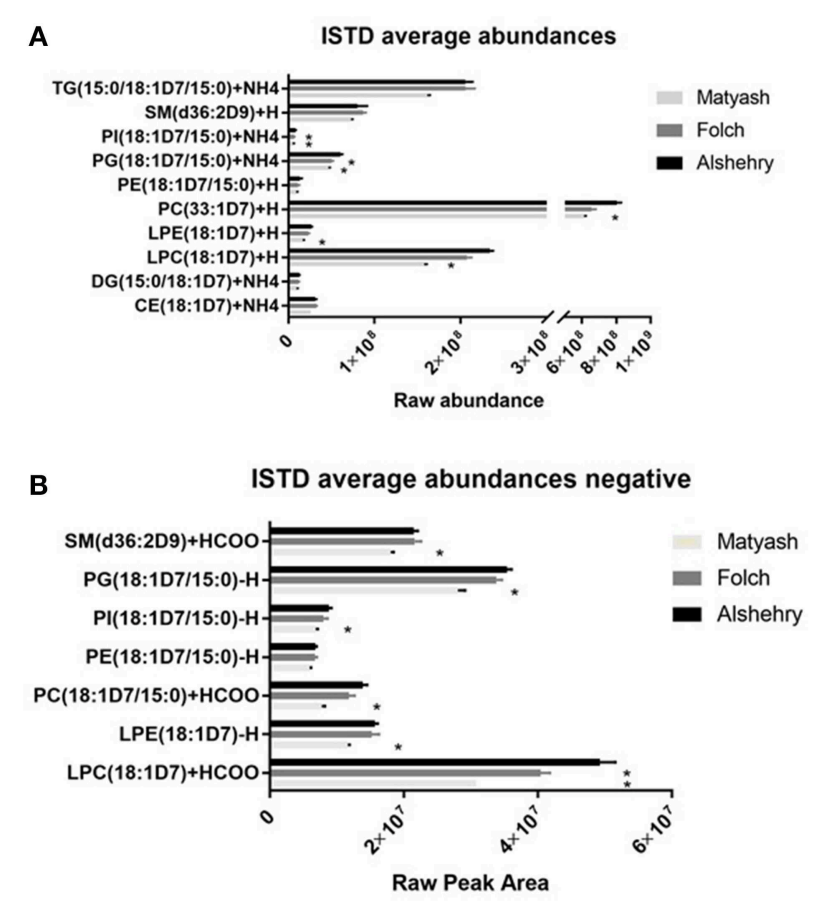

FIGURE 3 | ISTD raw abundances across the three extraction methods in (A) positive ion mode and (B) negative ion mode. The Alshehry method was as effective as the Folch and Matyash method in extracting most lipid classes and was more effective in extraction of more polar lipids (LPC, LPE and PI). Error bars represent $\mathrm{SE}\left({ }^{*} P<0.05\right.$ relative to single phase method). CE, cholesterol ester; D5DG, deuterated diacylglycerol; DG, diacylglycerol; LPC,

lysophosphatidylcholine; LPE, Iysophosphatidylethanolamine; PC,

phosphatidylcholine; PE, phosphatidylethanolamine; PG, phosphatidylglycerol, PI, phosphatidylinositol, PS, phosphatidylserine, SM, sphingomyelin; TG, triacylglycerol.

\section{Reproducibility of Methods}

We also compared intra-assay coefficients of variation (Figure 5), defined as the standard deviation of the peak areas across several samples in a single experimental batch divided by the mean of the peak area, expressed as a percentage. A lower CV\% then corresponds to consistency of values obtained from extraction of technical replicates. The median intra-assay (repeat injections within a single experiment) and inter-assay (injections from samples processed across separate days) coefficients of variation $(\mathrm{CV} \%)$ for all lipids in positive ion mode originally reported by Alshehry were 12 and 14\% respectively (37). Here, we report median intra-assay and inter-assay CV of 14.2 and $14.4 \%$, respectively for the Alshehry extraction, which is consistent with this previous report. The median intra-assay CV\% we obtained using the Alshehry method was lower compared to that obtained for the Matyash and Folch methods (21.8 and 15.1\% respectively, $p<0.0001$ and $p=0.014$ respectively, Mann-Whitney $U$ test). Seventy-five percent of lipids in the Alshehry method had a CV of $21 \%$ or lower, and the median CV for internal standards was 8.5\%. Median CV for lipids in negative mode were 12.7, 17.5, and $12.2 \%$ for the Alshehry, Matyash and Folch recipes respectively $(p<0.0001$ for Folch and Alshehry relative to Matyash,
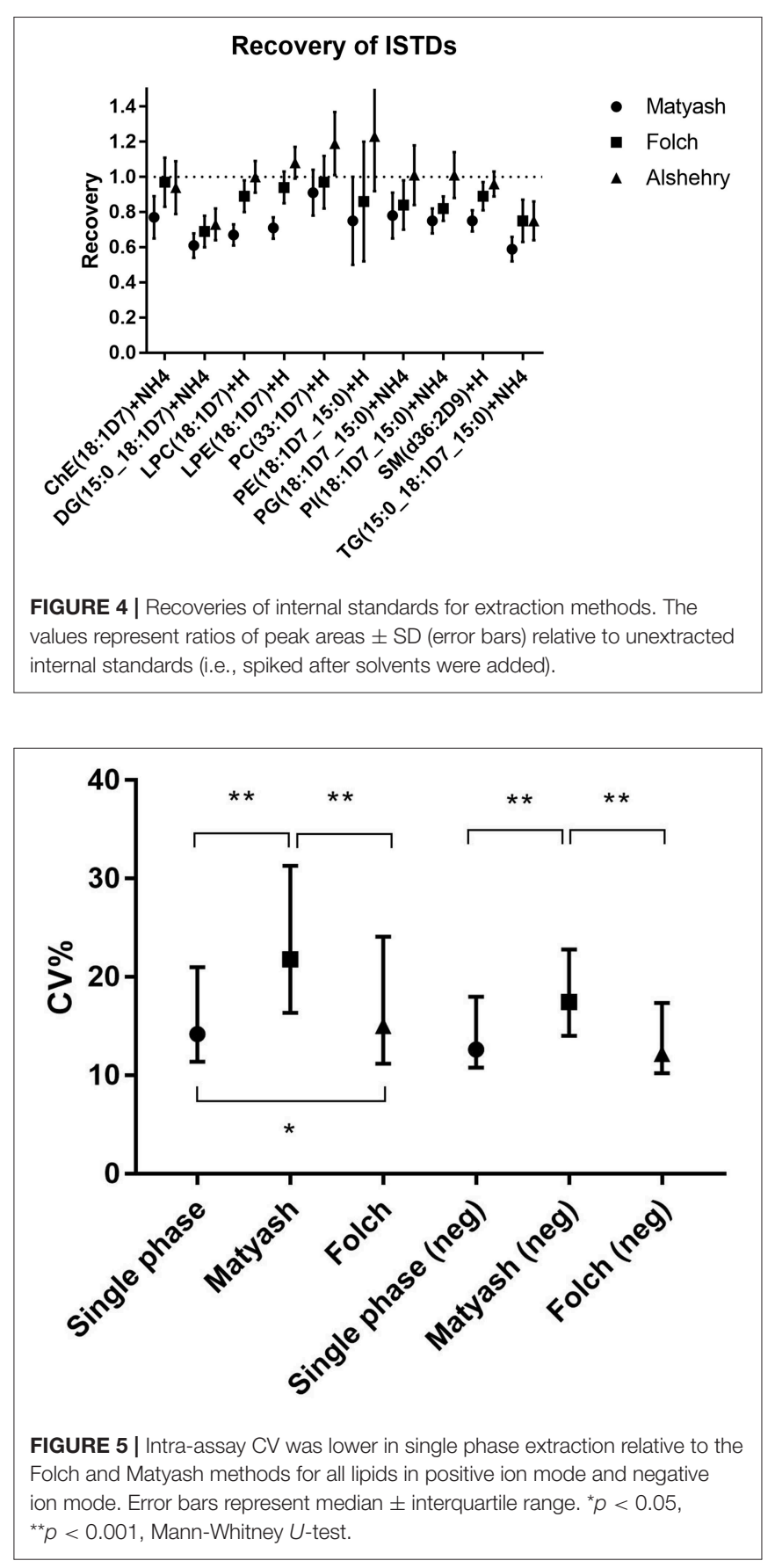

Mann-Whitney $U$-test). The comparative data collected in this experiment is summarized in Table 1. Typical representative total ion chromatograms of internal standards (Figure 6A), alongside extracted ion chromatograms for each standard (Figure 6B), as well as the total ion chromatogram for plasma lipids (Figure 6C) are shown.

\section{DISCUSSION}

In the original study by Alshehry et al. (25) the single phase 1-butanol/methanol 1:1 (v/v) method was reported to be as 
good an extraction as similar single phase extraction protocols with lipid measurements (based on peak areas normalized against internal standards). In particular, results between the Alshehry method and a single phase 2:1 methanol/chloroform

TABLE 1 | Results summary.

\begin{tabular}{lccc}
\hline & Matyash & Folch & Alshehry \\
\hline $\begin{array}{l}\text { Volume of plasma per sample (this } \\
\text { study) }\end{array}$ & $10 \mu \mathrm{L}$ & $10 \mu \mathrm{L}$ & $10 \mu \mathrm{L}$ \\
$\begin{array}{l}\text { Volume of plasma per sample } \\
\text { (original) }\end{array}$ & $200 \mu \mathrm{L}$ & $200 \mu \mathrm{L}$ & $10 \mu \mathrm{L}$ \\
$\begin{array}{l}\text { Volume of solvents per sample (this } \\
\text { study) }\end{array}$ & $900 \mu \mathrm{L}$ & $480 \mu \mathrm{L}$ & $100 \mu \mathrm{L}$ \\
$\begin{array}{l}\text { Volume of solvents per sample } \\
\text { (original) }\end{array}$ & $18,000 \mu \mathrm{L}$ & $9,600 \mu \mathrm{L}$ & - \\
$\begin{array}{l}\text { Numbers of individual lipids in positive } \\
\text { ion mode }\end{array}$ & 381 & 400 & 390 \\
$\begin{array}{l}\text { Numbers of individual lipids in } \\
\text { negative ion mode }\end{array}$ & 122 & 127 & 119 \\
$\begin{array}{l}\text { Median intra-assay CV\% in positive } \\
\text { ion mode } \\
\text { Median intra-assay CV\% in negative }\end{array}$ & 21.8 & 15.1 & 14.1 \\
$\begin{array}{l}\text { ion mode } \\
\text { Average total recovery \% }\end{array}$ & 17.5 & 12.2 & 12.7 \\
& 73 & 86 & 99
\end{tabular}

extraction method were strongly correlated $\left(r^{2}=0.976\right)$. In the present study, both the Matyash and Folch methods were also strongly correlated with Alshehry method $(r=0.97$ and 0.99 , respectively) which suggests strong agreeance between the methods, also supported by Bland-Altman bias ratios. Overall, this suggests that methods correspond well, though the Alshehry method may be superior in detection of more lowly abundant lipid species. Number of extracted lipids were also similar between the methods indicating similar coverage of lipids for all three extractions.

Although most lipids classes were extracted similarly between methods, certain lipid classes were more abundantly extracted using the Alshehry method as opposed to the Matyash or Folch methods. This included the lysophospholipids LPC and LPE, as well as some glycerophospholipids containing a polar head group, such as PC, PI and PG. This is consistent with reports that the Alshehry method is more amenable to extraction of highly polar lipids compared to other methods (25), since the hydrophilic phases are discarded in these biphasic methods. A previous pilot analysis also showed markedly greater extraction of the highly polar lipid standards, phosphatidylserine, PS(17:0/17:0) and sphingosine phosphate, SoP(d17:1) in the Alshehry method compared to either the Matyash or Folch methods. The ability of the Alshehry method to capture more polar lipids may be particularly useful in analysis of
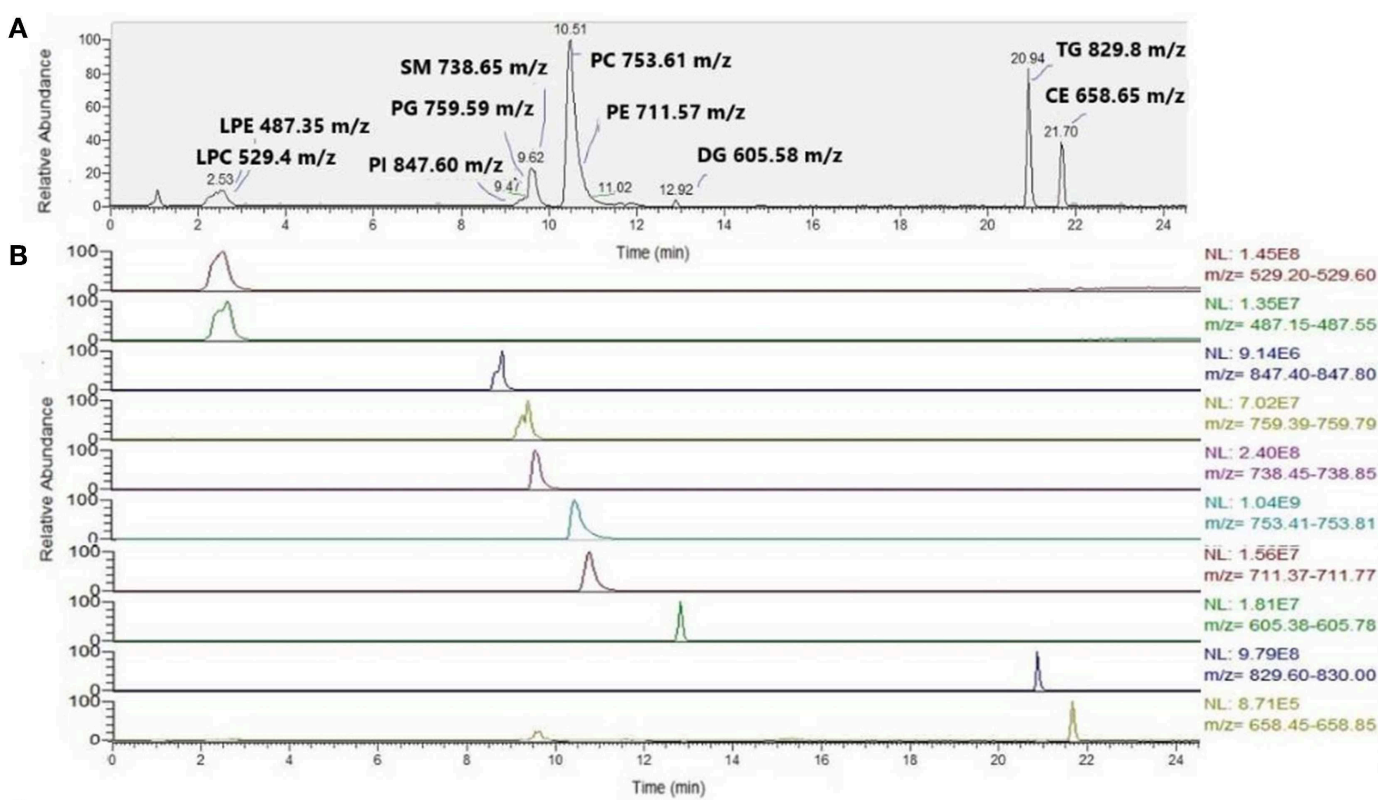

C

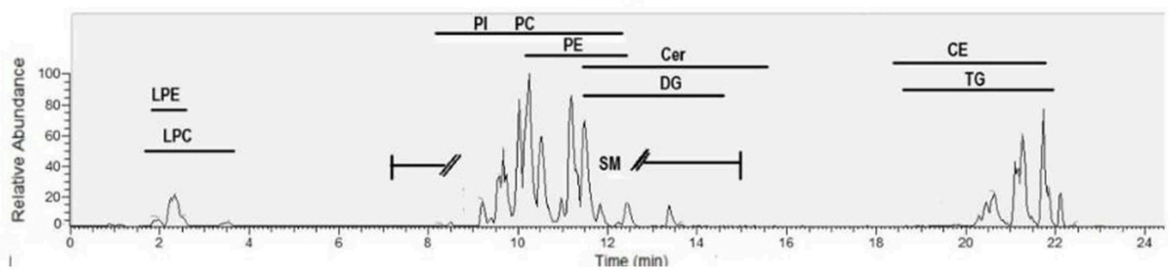

FIGURE 6 | Typical chromatogram featuring (A) SPLASH Lipidomix internal standards in positive ion mode (total ion chromatogram), (B) SPLASH Lipidomix internal standards (extracted ion chromatograms for each standard narrowed to $\pm 0.2 \mathrm{Da}$ of the theoretical $\mathrm{m} / \mathrm{z}$ ), presented from top to bottom in order of increasing retention time, and (C) corresponding plasma sample in positive ion mode. Typical retention time ranges of elution (minutes) are provided for various lipid classes. 
neuropsychiatric disorders and dementia where these lipids have been implicated in these conditions $(17,38,39)$.

Although the Alshehry recipe is ideal for extracting more polar lipids, the method is adaptable for extraction of other lipid classes. For example, a similar study of lipid extraction in cow's milk applied a modified version of the Alshehry single phase method in order to extract higher levels of non-polar lipids (40). This modified version used a single phase mixture of 1butanol/methanol/chloroform (3:5:4 v:v:v) in order to broaden the coverage of highly lipophilic species, especially triglycerides. Other similar single-phase extraction procedures using different solvent ratios have been described for lipid extraction, such as a 3:1 butanol/methanol extraction for plasma (41) and animal tissue (42), as well as a methanol/MTBE/chloroform (1.33/1/1 v:v:v) extraction $(43,44)$. All these cases, report comparable, if not greater yields against the Matyash and Folch methods suggesting good feasibility of single-phase extraction methods against traditional biphasic methods, as well as adaptability to capture a wide range of lipids of interest.

Overall, there was strong recovery for a large majority of lipids with the single-phase method, at $99 \%$, especially for polar lipids. The high recovery of the Alshehry method near $100 \%$ is consistent with the fact that this method does not experience the risk of dripping losses that occurs with the other two biphasic methods as the bulk of lipids are present in a single phase to handle and readily removable for lipid analysis.

The less than optimal recovery (73\%) for the Matyash method compared to previously reported is likely related to the relatively low volume of solvent used during the 20 -fold volume scale down procedure, as well as the tendency of MTBE to evaporate quickly, affecting extraction reproducibility (41), which is also reflected in the higher CV\% (see below).

Analysis of reproducibility based on $\mathrm{CV} \%$ revealed the Alshehry method had a median CV\% of $14.2 \%$, lower than or equal to the Folch method, and even better than the Matyash method (at 15.1 and $21.8 \%$ respectively, in positive ion mode). Thus, the Alshehry protocol produces reliable lipid measurements over technical replicates within experimental batches as well as, or even better than the established Folch and Matyash approaches, which is expected considering reduced dripping losses in a monophasic extraction. Additionally, the inter-assay CV\% is very similar to that of the intra-assay CV\% for the Alshehry method which suggests that the method produces reliable measurements across experimental batches and any additional variation to this method due to batch differences would be minimal.

Furthermore, the single phase Alshehry method is advantageous in that lipids are drawn into a single phase and can be easily removed from the sample as supernatant without picking up non-extractable matrix. This makes the method easier and potentially faster compared to conventional two-phase solvent partition systems where there is also an increased risk of contamination from unwanted analytes, and the risk of losses during transfer between phases. Additionally, the method can be applied to as little as $10 \mu \mathrm{l}$ of plasma, and using just $100 \mu \mathrm{l}$ of solvent per sample, and therefore greatly minimizes the amount of sample and solvent required for extraction. By contrast, the original Matyash extraction reports extractions using $200 \mu \mathrm{l}$ of plasma, and requires $900 \mu \mathrm{l}$ of MTBE and methanol combined pre-scale down, which translates to $18 \mathrm{ml}$ of solvent required per plasma sample in the original Matyash method. Considering the broad range of lipids and reduced losses reflected in the high degree of recovery and reproducibility obtainable using a relatively small volume of sample and solvent in the Alshehry method, this represents a strong advantage of the method over existing biphasic methods. The volume savings can be particularly important for high value plasma samples from expensive clinical and population-based studies, since such samples are a finite resource and conservative usage is necessary, to maximize potential experiments and makes extraction of many samples much simpler to handle.

This new method has already been applied for lipid extraction of plasma from large populations for the study of health and disease $(37,45)$. Nevertheless, we highlight one important limitation of the Alshehry method as reported by the original authors (25): due to the monophasic nature of extraction, ionic contaminants are not readily removed during extraction, leading to an increased risk of ion suppression effects. The Alshehry method is therefore unsuitable for shotgun/direct infusion lipidomics techniques, but is suitable for use with reverse phase LC-ESI MS/MS.

It needs acknowledging that in context of neuropsychiatric disorders and dementia, it is important to establish the connection between putative changes in peripheral signals with that of signals generated by the central nervous system (CNS). Despite all the advantages associated with peripheral biomarkers, a limitation of plasma-based lipidomics research is that the blood brain barrier (BBB) restricts passage of metabolites between the CSF and blood compartments. Therefore, there is not always a strong correspondence between blood and centrally derived metabolites. Nevertheless, many CSF metabolites are selectively reabsorbed back into the vascular system, and essential lipids such as polyunsaturated fatty acids are transported to the brain from blood as free fatty acids (46) or as part of phospholipids, such as lysophosphatidylcholine (47), a lipid class well-detected using the novel method. The BBB may have increased permeability in response to inflammation or disease states $(48,49)$. Moreover, there is substantial overlap in metabolic changes identified between matched CSF and plasma $(50,51)$, and some blood lipids may be correlated with CSF tau and amyloid load (52), as well as other imaging measures such as brain atrophy (53).

Other studies have also identified relationships between plasma/serum lipids, apolipoproteins and cognition $(54,55)$. A recent study identified differential impacts of apolipoprotein $\mathrm{E}$ in the CNS and periphery, finding deficits in apolipoprotein $\mathrm{E}$ in the CNS induced synaptic loss, whereas a deficit in the periphery lead to impaired cognition (56), though synaptic loss could partially be rescued by increasing plasma apolipoprotein E levels. The potential of plasma lipids to predict memory performance and cognitive decline (or protection) several years in advance (57-59) presents evidence that these lipids may have a substantial influence on the CNS, or that plasma lipids may reflect some underlying pathological process 
occurring in the CNS. Many of the metabolites reported in the above studies are robustly extracted using the novel Alshehry method, such as sphingolipids, PC, PE and their corresponding lysophospholipids, and have also similarly been found to be altered in post-mortem brain tissue or CSF in patients with Alzheimer's disease $(38,50,60)$. We have previously reviewed plasma lipids thought to be altered in Alzheimer's disease (18), which includes plasmalogen phospholipids, sphingomyelins and ceramides. Further, a recent untargeted lipidomics study in plasma found substantial differences in the lipidomic profile between healthy controls, Alzheimer's disease and behavioral variant frontotemporal dementia (bvFTD) (19). Of note, the authors were able to confirm hypertriglyceridemia in bvFTD at the lipid species level, and also detected altered levels of dietary lipids thought to characterize binge eating behaviors among these patients. This study, among others demonstrates the utility of exploring plasma lipids as potential biomarkers for neuropsychiatric and neurological disorders. Even with such a wealth of studies, independent validation and replication remains a high priority concern in the lipid biomarker research community (18). Further, the natural variation in lipids within and between individuals independent of disease is still being characterized $(45,61,62)$, which is an important factor to consider before applying comparative lipidomics in disease. Although this pilot study does not focus on inter-individual differences in lipids, we have recently applied this particular method in a larger study of 100 individuals to identify the natural variation of the plasma lipidome in cognitively healthy individuals by age, sex and BMI (63). For future studies, we intend to investigate the influence of $A P O E$ genotype on the plasma lipidome, as well as identify lipidomic changes associated with vascular dementia. We believe that a consistent and simple method for plasma lipid extraction, such as explored in this paper, will go a long way toward expanding blood lipidomic studies, give due insight into neurological processes and minimize some of the variance in literature of reported biomarkers inherent in extraction methodology.

\section{CONCLUSION}

Overall, we confirm the findings of the originally published Alshehry single-phase protocol, but also show that it is good as, if not better than the commonly used biphasic Matyash and Folch methods in the general extraction of plasma lipids, based on its ability to cover a broad range of lipid classes, including more polar lipids that may not be extracted readily using the Folch recipe. The Alshehry method also provides reliable and reproducible measurements when lipids are analyzed by LC-MS/MS above that of the Folch and Matyash methods. The single-phase method offers a safe, environmentally friendly (chloroform-free) and economical method for extraction of plasma lipids with high (nearly 100\%) recovery that is a good substitute for the traditional approaches. Notably, all methods produce highly correlated results, indicating that reliable data can be obtained with all these protocols. However, the simplicity involved in having most lipids extractable in a single phase and low plasma and solvent volumes required by this Alshehry method may be particularly useful where sample volumes are limited, for projects where high sample numbers need to be assayed for lipidomic analysis, or where plasma needs to be conserved to maximize experiments.

\section{DATA AVAILABILITY}

The datasets generated for this study are available on request to the corresponding author.

\section{ETHICS STATEMENT}

The study was approved by the Ethics Committees of the University of New South Wales and the South Eastern Sydney and Illawarra Area Health Service (ethics approval HC12313 and $\mathrm{HC14327}$, respectively). All work involving human subjects conformed to the principles of the Declaration of Helsinki of the World Medical Association and both informed and written consent was obtained.

\section{AUTHOR CONTRIBUTIONS}

MW, NB, and AP wrote and drafted the manuscript. NB, RP, AP, and PS conceived of the project. MW performed the experiments and analyzed the data. RP provided technical expertise and training for use of the QExactive Plus mass spectrometer and LipidSearch software.

\section{FUNDING}

This work was supported by a National Health \& Medical Research Council of Australia Program Grant (APP1054544). MW was the recipient of an Australian Postgraduate Award (APA) from the Australian Commonwealth Government. NB was the recipient of the Australian Research Council Postdoctoral Research Fellowship. The authors thank the Rebecca Cooper Medical Research Foundation for their ongoing financial support.

\section{ACKNOWLEDGMENTS}

The authors would like to acknowledge the contributions of Dr. Kristan Kang and Mahboobeh Hosseini, who provided data collection and biobanking for plasma samples, as well as Ms. Angela Russell and Dr. Sophia Dean, who provided administrative support. The authors also thank the Mark Wainwright Analytical Center in conjunction with the Bioanalytical Mass Spectrometry Facility for providing use of the QExactive mass spectrometer, analytical grade reagents and data processing software. The authors would also like to thank CHeBA and SEALs Pathology (Prince of Wales Hospital) for handling and preparing of the MAS samples, as well as the MAS research teams and participants for making this project possible. 


\section{REFERENCES}

1. Nielsen MM, Lambertsen KL, Clausen BH, Meyer M, Bhandari DR, Larsen ST, et al. Mass spectrometry imaging of biomarker lipids for phagocytosis and signalling during focal cerebral ischaemia. Sci Rep. (2016) 6:39571. doi: 10.1038/srep39571

2. Wu W, Shi X, Xu C. Regulation of T cell signalling by membrane lipids. Nat Rev Immunol. (2018) 18:219. doi: 10.1038/nri.2018.9

3. Degli Esposti M. The mitochondrial battlefield and membrane lipids during cell death signalling. Ital J Biochem. (2003) 52:43-50.

4. Nadler A, Yushchenko DA, Muller R, Stein F, Feng S, Mulle C, et al. Exclusive photorelease of signalling lipids at the plasma membrane. Nat Commun. (2015) 6:10056. doi: 10.1038/ncomms10056

5. Davison AN. Biosynthesis of the myelin sheath. In: Lipids, Malnutrition and The Developing Brain. Netherlands: Ciba Foundation Symposium (1971). p. 73-90.

6. Igarashi M, Suzuki K. Effect of exogenous lipids on activites on the rat brain cholesterol ester hydrolase localized in the myelin sheath. J Neurochem. (1976) 27:859-66. doi: 10.1111/j.1471-4159.1976.tb05147.x

7. Bazan NG, Packard MG, Teather L, Allan G. Bioactive lipids in excitatory neurotransmission and neuronal plasticity. Neurochem Int. (1997) 30:225-31. doi: 10.1016/S0197-0186(96)00020-4

8. Haro A, Saxlin T, Suominen AL, Ylostalo P, Leiviska J, Tervonen T, et al. Serum lipids modify periodontal infection - C-reactive protein association. J Clin Periodontol. (2012) 39:817-23. doi: 10.1111/j.1600-051X.2012.01920.x

9. Ulas F, Balbaba M, Ozmen S, Celebi S, Dogan U. Association of dehydroepiandrosterone sulfate, serum lipids, C-reactive protein and body mass index with age-related macular degeneration. Int Ophthalmol. (2013) 33:485-91. doi: 10.1007/s10792-013-9728-4

10. Schneider C. Lipids in health and disease. Mol Nutr Food Res. (2010) 54(Suppl. 1):S5. doi: 10.1002/mnfr.201090016

11. Braverman NE, Moser AB. Functions of plasmalogen lipids in health and disease. Biochim Biophys Acta. (2012) 1822:1442-52. doi: 10.1016/j.bbadis.2012.05.008

12. Finkelstein J, Heemels MT, Shadan S, Weiss U. Lipids in health and disease. Nature. (2014) 510:47. doi: 10.1038/510047a

13. Tawakol A, Jaffer F. Imaging the intersection of oxidative stress, lipids, and inflammation: progress toward personalized care of atherosclerosis. J Am Coll Cardiol. (2018) 71:336-8. doi: 10.1016/j.jacc.2017.11.031

14. Krishnan E. Inflammation, oxidative stress and lipids: the risk triad for atherosclerosis in gout. Rheumatology. (2010) 49:1229-38. doi: 10.1093/rheumatology/keq037

15. Sethi S, Brietzke E. Recent advances in lipidomics: analytical and clinical perspectives. Prostaglandins Other Lipid Mediat. (2017) 128-9:8-16. doi: 10.1016/j.prostaglandins.2016.12.002

16. Rustam YH, Reid GE. Analytical challenges and recent advances in mass spectrometry based lipidomics. Anal Chem. (2018) 90:374-97. doi: 10.1021/acs.analchem.7b04836

17. Gonzalez-Dominguez R, Garcia-Barrera T, Gomez-Ariza JL. Combination of metabolomic and phospholipid-profiling approaches for the study of Alzheimer's disease. J Proteomics. (2014) 104:37-47. doi: 10.1016/j.jprot.2014.01.014

18. Wong MW, Braidy N, Poljak A, Pickford R, Thambisetty M, Sachdev PS. Dysregulation of lipids in Alzheimer's disease and their role as potential biomarkers. Alzheimer Demen. (2017) 13:810-27. doi: 10.1016/j.jalz.2017.01.008

19. Kim WS, Jary E, Pickford R, He Y, Ahmed RM, Piguet O, et al. Lipidomics analysis of behavioral variant frontotemporal dementia: a scope for biomarker development. Front Neurol. (2018) 9:104. doi: 10.3389/fneur.2018.00104

20. Schneider M, Levant B, Reichel M, Gulbins E, Kornhuber J, Müller CP. Lipids in psychiatric disorders and preventive medicine. Neurosci Biobehav Rev. (2017) 76:336-62. doi: 10.1016/j.neubiorev.2016.06.002

21. Yang X, Sun L, Zhao A, Hu X, Qing Y, Jiang J, et al. Serum fatty acid patterns in patients with schizophrenia: a targeted metabonomics study. Translational Psychiatry. (2017) 7:e1176. doi: 10.1038/tp.2017.152

22. Reis A, Rudnitskaya A, Blackburn GJ, Mohd Fauzi N, Pitt AR, Spickett CM. A comparison of five lipid extraction solvent systems for lipidomic studies of human LDL. J Lipid Res. (2013) 54:1812-24. doi: 10.1194/jlr.M034330
23. Folch J, Lees M, Sloane Stanley GH. A simple method for the isolation and purification of total lipides from animal tissues. J Biol Chem. (1957) 226:497509.

24. Matyash V, Liebisch G, Kurzchalia TV, Shevchenko A, Schwudke D. Lipid extraction by methyl-tert-butyl ether for high-throughput lipidomics. J Lipid Res. (2008) 49:1137-46. doi: 10.1194/jlr.D700041-JLR200

25. Alshehry ZH, Barlow CK, Weir JM, Zhou Y, McConville MJ, Meikle PJ. An efficient single phase method for the extraction of plasma lipids. Metabolites. (2015) 5:389-403. doi: 10.3390/metabo5020389

26. Alshehry ZH, Meikle PJ. Liquid extraction: single-phase butanol/methanol extraction. In: Wenk MR, editor. Encyclopedia of Lipidomics. Dordrecht:Springer (2016). p. 1-4.

27. Welsh P, Hart C, Papacosta O, Preiss D, McConnachie A, Murray $\mathrm{H}$, et al. Prediction of cardiovascular disease risk by cardiac biomarkers in 2 United Kingdom cohort studies: does utility depend on risk thresholds for treatment? Hypertension. (2016) 67:309-15. doi: 10.1161/HYPERTENSIONAHA.115.06501

28. Ulmer C, Jones CM, Yost R, Garrett T, Bowden J. Optimization of folch, bligh-dyer, and matyash sample-to-extraction solvent ratios for human plasma-based lipidomics studies. Anal Chim Acta. (2018) 1037:351-7. doi: 10.1016/j.aca.2018.08.004

29. Wang M, Wang C, Han X. Selection of internal standards for accurate quantification of complex lipid species in biological extracts by electrospray ionization mass spectrometry-What, how and why? Mass Spectrom Rev. (2016) 36:693-714. doi: 10.1002/mas.21492

30. Sachdev PS, Brodaty H, Reppermund S, Kochan NA, Trollor JN, Draper B, et al. The Sydney memory and ageing study (MAS): methodology and baseline medical and neuropsychiatric characteristics of an elderly epidemiological non-demented cohort of Australians aged 70-90 years. Int Psychogeriatr. (2010) 22:1248-64. doi: 10.1017/S1041610210001067

31. Tsang RS, Sachdev PS, Reppermund S, Kochan NA, Kang K, Crawford J, et al. Sydney memory and ageing study: an epidemiological cohort study of brain ageing and dementia. Int Rev Psychiatry. (2013) 25:711-25. doi: 10.3109/09540261.2013.860890

32. Winblad B, Palmer K, Kivipelto M, Jelic V, Fratiglioni L, Wahlund LO, et al. Mild cognitive impairment-beyond controversies, towards a consensus: report of the international working group on mild cognitive impairment. $J$ Intern Med. (2004) 256:240-6. doi: 10.1111/j.1365-2796.2004.01380.x

33. Connors MH, Sachdev PS, Kochan NA, Xu J, Draper B, Brodaty H. Cognition and mortality in older people: the Sydney memory and ageing study. Age Ageing. (2015) 44:1049-54. doi: 10.1093/ageing/afv139

34. O’Bryant SE, Gupta V, Henriksen K, Edwards M, Jeromin A, Lista S, et al. Guidelines for the standardization of preanalytic variables for blood-based biomarker studies in Alzheimer's disease research. Alzheimers Dement. (2015) 11:549-60. doi: 10.1016/j.jalz.2014.08.099

35. Mielke MM, Bandaru VV, Han D, An Y, Resnick SM, Ferrucci L, et al. Factors affecting longitudinal trajectories of plasma sphingomyelins: the Baltimore Longitudinal Study of Aging. Aging Cell. (2015) 14:112-21. doi: 10.1111/acel.12275

36. Burla B, Arita M, Arita M, Bendt AK, Cazenave-Gassiot A, Dennis EA, et al. MS-based lipidomics of human blood plasma: a community-initiated position paper to develop accepted guidelines. J Lipid Res. (2018) 59:2001-17. doi: $10.1194 /$ jlr.S087163

37. Alshehry ZH, Mundra PA, Barlow CK, Mellett NA, Wong G, McConville $\mathrm{MJ}$, et al. Plasma lipidomic profiles improve on traditional risk factors for the prediction of cardiovascular events in type 2 diabetes mellitus. Circulation. (2016) 134:1637-50. doi: 10.1161/CIRCULATIONAHA.116. 023233

38. Han X, Holtzman DM, McKeel DWJr. Plasmalogen deficiency in early Alzheimer's disease subjects and in animal models: molecular characterization using electrospray ionization mass spectrometry. J Neurochem. (2001) 77:1168-80. doi: 10.1046/j.1471-4159.2001.00332.x

39. Kosicek M, Hecimovic S. Phospholipids and Alzheimer's disease: alterations, mechanisms and potential biomarkers. Int J Mol Sci. (2013) 14:1310-22. doi: 10.3390/ijms14011310

40. Liu Z, Rochfort S, Cocks BG. Optimization of a single phase method for lipid extraction from milk. J Chromatogr A. (2016) 1458:145-9. doi: $10.1016 /$ j.chroma.2016.06.055 
41. Lofgren L, Stahlman M, Forsberg GB, Saarinen S, Nilsson R, Hansson GI. The BUME method: a novel automated chloroform-free 96-well total lipid extraction method for blood plasma. J Lipid Res. (2012) 53:1690-700. doi: 10.1194/jlr.D023036

42. Lofgren L, Forsberg GB, Stahlman M. The BUME method: a new rapid and simple chloroform-free method for total lipid extraction of animal tissue. Sci Rep. (2016) 6:27688. doi: 10.1038/srep27688

43. Pellegrino RM, Di Veroli A, Valeri A, Goracci L, Cruciani G. LC/MS lipid profiling from human serum: a new method for global lipid extraction. Anal Bioanal Chem. (2014) 406:7937-48. doi: 10.1007/s00216-014-8255-0

44. Gil A, Zhang W, Wolters JC, Permentier H, Boer T, Horvatovich P, et al. One- vs two-phase extraction: re-evaluation of sample preparation procedures for untargeted lipidomics in plasma samples. Anal Bioanal Chem. (2018) 410:5859-70. doi: 10.1007/s00216-018-1200-x

45. Saw W-Y, Tantoso E, Begum H, Zhou L, Zou R, He C, et al. Establishing multiple omics baselines for three Southeast Asian populations in the Singapore integrative omics study. Nat Commun. (2017) 8:653. doi: 10.1038/s41467-017-00413-x

46. Freund Levi Y, Vedin I, Cederholm T, Basun H, Faxen Irving G, Eriksdotter M, et al. Transfer of omega-3 fatty acids across the blood-brain barrier after dietary supplementation with a docosahexaenoic acid-rich omega-3 fatty acid preparation in patients with Alzheimer's disease: the OmegAD study. J Intern Med. (2014) 275:428-36. doi: 10.1111/joim.12166

47. Nguyen LN, Ma D, Shui G, Wong P, Cazenave-Gassiot A, Zhang X, et al. Mfsd2a is a transporter for the essential omega- 3 fatty acid docosahexaenoic acid. Nature. (2014) 509:503-6. doi: 10.1038/nature13241

48. Marques F, Sousa JC, Sousa N, Palha JA. Blood-brain-barriers in aging and in Alzheimer's disease. Mol Neurodegener. (2013) 8:38. doi: 10.1186/1750-1326-8-38

49. Marottoli FM, Katsumata Y, Koster KP, Thomas R, Fardo DW, Tai LM. Peripheral inflammation, apolipoprotein E4, and amyloid-beta interact to induce cognitive and cerebrovascular dysfunction. ASN Neuro. (2017) 9:1759091417719201. doi: 10.1177/1759091417719201

50. Trushina E, Dutta TX,Persson MT, Mielke MM, Petersen RC. identification of altered metabolic pathways in plasma and CSF in mild cognitive impairment and Alzheimer's disease using metabolomics. PLoS ONE. (2013) 8:e63644. doi: 10.1371/journal.pone.0063644

51. Cho SM, Kim HV, Lee S, Kim HY, Kim W, Kim TS, et al. Correlations of amyloid- $\beta$ concentrations between CSF and plasma in acute Alzheimer mouse model. Sci Reports. (2014) 4:6777. doi: 10.1038/srep06777

52. Chatterjee P, Lim WL, Shui G, Gupta VB, James I, Fagan AM, et al. Plasma phospholipid and sphingolipid alterations in presenilin1 mutation carriers: a pilot study. J Alzheimers Dis. (2016) 50:887-94. doi: 10.3233/JAD-150948

53. Proitsi P, Kim M, Whiley L, Simmons A, Sattlecker M, Velayudhan L, et al. Association of blood lipids with Alzheimer's disease: a comprehensive lipidomics analysis. Alzheimers Demen. (2016) 13:140-51. doi: 10.1016/j.jalz.2016.08.003

54. Song F, Poljak A, Crawford J, Kochan NA, Wen W, Cameron B, et al. Plasma apolipoprotein levels are associated with cognitive status and decline in a community cohort of older individuals. PLoS ONE. (2012) 7:e34078 doi: 10.1371/journal.pone.0034078

55. Muenchhoff J, Song F, Poljak A, Crawford JD, Mather KA, Kochan NA, et al. Plasma apolipoproteins and physical and cognitive health in very old individuals. Neurobiol Aging. (2017) 55:49-60. doi: 10.1016/j.neurobiolaging.2017.02.017

56. Lane-Donovan C, Wong WM, Durakoglugil MS, Wasser CR, Jiang S, Xian $\mathrm{X}$, et al. Genetic restoration of plasma ApoE improves cognition and partially restores synaptic defects in ApoE-deficient mice. J Neurosci. (2016) 36:1014150. doi: 10.1523/JNEUROSCI.1054-16.2016

57. Mielke MM, Bandaru VV, Haughey NJ, Xia J, Fried LP, Yasar S, et al. Serum ceramides increase the risk of Alzheimer disease: the women's health and aging study II. Neurology. (2012) 79:633-41. doi: 10.1212/WNL.0b013e318264e380

58. Mapstone M, Cheema AK, Fiandaca MS, Zhong X, Mhyre TR, MacArthur LH, et al. Plasma phospholipids identify antecedent memory impairment in older adults. Nat Med. (2014) 20:415-8. doi: 10.1038/nm.3466

59. Fiandaca MS, Zhong X, Cheema AK, Orquiza MH, Chidambaram S, Tan MT, et al. Plasma 24-metabolite panel predicts preclinical transition to clinical stages of Alzheimer's disease. Front Neurol. (2015) 6:237. doi: 10.3389/fneur.2015.00237

60. Pettegrew JW, Panchalingam K, Hamilton RL, McClure RJ. Brain membrane phospholipid alterations in Alzheimer's disease. Neurochem Res. (2001) 26:771-82. doi: 10.1023/A:1011603916962

61. Begum H, Li B, Shui G, Cazenave-Gassiot A, Soong R, Ong RT, et al. Discovering and validating between-subject variations in plasma lipids in healthy subjects. Sci Rep. (2016) 6:19139. doi: 10.1038/srep 19139

62. Begum H, Torta F, Narayanaswamy P, Mundra PA, Ji S, Bendt AK, et al. Lipidomic profiling of plasma in a healthy Singaporean population to identify ethnic specific differences in lipid levels and associations with disease risk factors. Clin Mass Spectrom. (2017) 6:25-31. doi: 10.1016/j.clinms.201 7.11.002

63. Wong MWK, Braidy N, Pickford R, Vafaee F, Crawford J, Muenchhoff J, et al. Plasma lipidome variation during the second half of the human lifespan is associated with age and sex but minimally with BMI. PLoS ONE. (2019) 14:e0214141. doi: 10.1371/journal.pone.0214141

Conflict of Interest Statement: The authors declare that the research was conducted in the absence of any commercial or financial relationships that could be construed as a potential conflict of interest.

Copyright (c) 2019 Wong, Braidy, Pickford, Sachdev and Poljak. This is an openaccess article distributed under the terms of the Creative Commons Attribution License (CC BY). The use, distribution or reproduction in other forums is permitted, provided the original author(s) and the copyright owner(s) are credited and that the original publication in this journal is cited, in accordance with accepted academic practice. No use, distribution or reproduction is permitted which does not comply with these terms. 